\title{
Transformation of the acute myeloid leukemia to acute B cell lymphoblastic leukemia with CAR-T cell therapy: a case report and review of the literature
}

\author{
Yanan Zhang ${ }^{1}$, Fengan $\mathrm{Liu}^{1}$, Xue Wang ${ }^{1}$, Jiang Cao ${ }^{1}$, Wei Chen ${ }^{1}$, Kunming $\mathrm{Qi}^{1}$, Kailin \\ $\mathrm{Xu}^{1}$, and Hai Cheng ${ }^{1}$ \\ ${ }^{1}$ The Affiliated Hospital of Xuzhou Medical University
}

November 11, 2021

\begin{abstract}
Lineage conversion is used to describe acute myeloid or lymphoid leukemia becomes the opposite at relapse.We report a 4-yearold child with acute myeloid leukemia who was converted to acute lymphoblastic leukemia at relapse and received chimeric antigen receptor $\mathrm{T}$ (cell) therapy for reference.

Transformation of the acute myeloid leukemia to acute B cell lymphoblastic leukemia with CAR-T cell therapy: a case report and review of the literature

Ya-nan Zhang ${ }^{1}$, Feng-an $\mathrm{Liu}^{1}$,Xue Wang ${ }^{1,2}$, Jiang $\mathrm{Cao}^{1,2}$, Wei Chen ${ }^{1,2}$, Kun-ming Qi ${ }^{1,2}$, Kai-lin Xu ${ }^{1,2,3}$, Hai Cheng ${ }^{1,2}$

1 Department of hematology, Affiliated Hospital of Xuzhou Medical University, Xuzhou 221002, Jiangsu Province, China

2 Blood Diseases Institute, Xuzhou Medical University, Xuzhou 221000, Jiangsu Province, China

3 Key Laboratory of Bone Marrow Stem Cell, Xuzhou 221000, Jiangsu Province, China

Abstract : Lineage conversion is used to describe acute leukemia that meets the FAB criteria for lymphoid or myeloid lineage at first but transforms at relapse. We report a 4-year-old child with acute myeloid leukemia (AML) who was converted to acute lymphoblastic leukemia (ALL) at relapse and received chimeric antigen receptor $\mathrm{T}$ (cell) therapy.

Keywords : acute leukemia, lineage switch, CAR-T therapy

\section{Correspondence:}

Hai Cheng

Department of hematology, Affiliated Hospital of Xuzhou Medical University, Xuzhou 221002, Jiangsu Province, China

Blood Diseases Institute, Xuzhou Medical University, Xuzhou 221000, Jiangsu Province, China

Email: hai_cheng@xzhmu.edu.cn
\end{abstract}

\section{Case presentation}


A 4-year-old female was admitted to the hospital because of fever with abdominal pain for 5 days. Abdominal computed tomography $(\mathrm{CT})$ showed a thick large intestinal shadow on the right side, with uneven density and flocculent high-density shadow near the fat space. The enhanced CT showed uneven strengthened right abdominal intestines, irregular thickening of the intestinal wall, and narrow lumen. Blood routine examination : white blood cells $(\mathrm{WBC}) 7.5 \times 10^{9} / \mathrm{L}, \mathrm{HGB} 88 \mathrm{~g} / \mathrm{L}, \mathrm{PLT} 31 \times 10^{9} / \mathrm{L}$. Bone marrow cytomorphologic examination : hyperplasia was active, granulocyte/erythroid ratio $(\mathrm{G} / \mathrm{E})=3.00 / 1$. Monoblast cells accounted for $9.5 \%$. Immature monocytes accounted for $31.5 \%$. Peroxidase, POX: weakly positive. Periodic Acid Schiff, PAS: negative. Flow cytometry analysis: the abnormal cells accounted for about $95.3 \%$ of nuclear cells and expressed HLA-DR, CD13, CD14, CD33, CD38, CD64, some cells expressed CD4, CD11b, CD15, CD56, MPO, a few cells expressed CD34. Chromosome karyotype analysis: 46, XY. Leukemia fusion gene screening: negative. The child was diagnosed with acute myeloid leukemia (AML-M5) according to FAB classification criteria.

Based on current guidelines for pediatric AML, this child received the standard induction therapy. The standard combination was $7+3$, with a 7 -day continuous infusion of cytarabine at the dosage of $100 \mathrm{mg} / \mathrm{m}^{2}$ per day on days 1 to 7 and daunorubicin at $60 \mathrm{mg} / \mathrm{m}^{2}$ per day on days 1 to 3 . Then nine courses of consolidation chemotherapy were followed. A monthly review of myelocytology and minimal residual disease(MRD) suggested that the primary disease maintained CR. At the 11th month after chemotherapy, the child's MRD indicated that the blast cells accounted for about $42.23 \%$ of the nuclear cells and expressed CD34, CD38 and partly expressed HLA-DR, CD19. Considering the possibility of recurrent leukemia, large-dose cytarabine combined with etoposide chemotherapy was given. Bone marrow cytomorphologic examination showed that immature monocytes accounted for $12 \%$ after chemotherapy and Idabisin combined with cytarabine (IA) regimen was given again. However, one month after applying IA regimen chemotherapy, bone marrow cytomorphologic examination shows that hyperplasia was active and lymphocytes are significantly increased (Figure 1). Bone marrow aspiration: POX staining: negative. PAS staining: positive. Flow cytometry analysis: blast cells accounted for about $89 \%$ of nuclear cells, mainly expressing HLA-DR, CD10, CD19, CD34, CD38, CD58, CD123, TdT. Chromosome karyotype analysis: 45, XX,-17 [6] / 46 XX [2]. In summary, the child has been converted to acute B lymphoblastic leukemia (B-ALL).

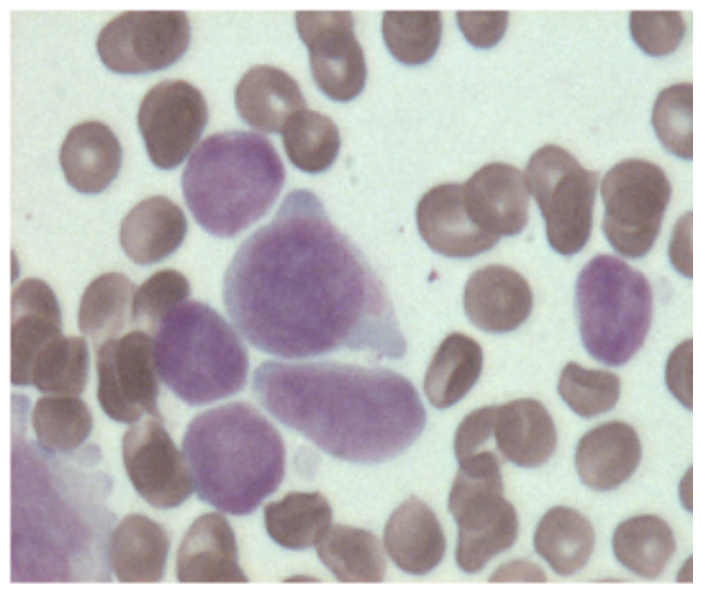

Figure 1: Figure 1 Bone marrow cytomorphologic examination shows that hyperplasia was active and lymphocytes are significantly increased

The child was pretreated with vinorelbine, daunorubicin and hydroprednisone (VDP) regimen and then injected with autologous anti-CD19- chimeric antigen receptor T cell (CAR-T cells) $1 \times 10^{6} / \mathrm{kg}$. The child developed recurrent high fever on day 10 after infusion of CAR-T cells, with body temperature up to 39.8 and the inflammatory factor of interleukin-6 (IL-6) and C-reactive protein (CRP) were significantly higher in plasma. At the same time, ferritin is also significantly increased (Figure 2). Cytokine release syndrome 
(CRS) after CAR-T treatment was considered. Meanwhile, the possibility of infection could not be ruled out. Antipyretic analgesics and anti-infective therapy were given and the body temperature was controlled one week later. No blast cells and immature lymphocytes were found in bone marrow on the 14th and 28th day after CAR-T cells infusion. Review of myelocytology once a month, indicating that the original disease to maintain CR. However, half a year later, bone marrow cytology showed that primitive lymphocytes accounted for $60 \%$, indicating that the primary disease relapsed. Cyclophosphamide, dexamethasone, vinorelbine and epirubicin (Hyper CVAD-A) chemo were given and autologous CAR-T cells were reinjected with $1 \times 10^{6} / \mathrm{kg}$. The child did not reach remission and later died of the progression of the disease.

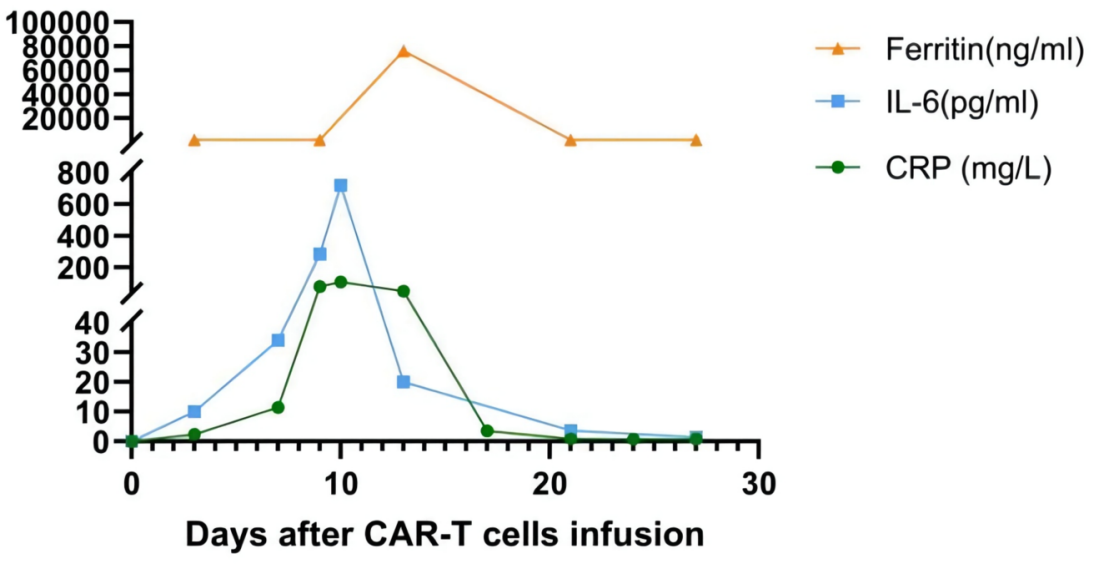

Figure 2: Figure 2 Inflammatory factor of interleukin-6 (IL-6) and C-reactive protein (CRP) and ferritin were significantly higher in plasma on the day 10 after infusion of CAR-T cells

\section{Discussion}

Leukemia is a kind of malignant clonal disease of hematopoietic stem and progenitor cells, which is characterized by uncontrolled proliferation of leukemic cells and inhibiting normal hematopoiesis. Although chemotherapy combined with hematopoietic stem cell transplantation (HSCT) has significantly improved the prognosis of patients with leukemia, a considerable number of patients still experience recurrence. Typically, recurrent leukemia patients have the same phenotype of blast cells in the bone marrow as initial diagnosis. However, some patients have lineage switch when the relapse occurs, that is, acute leukemia of one type (myeloid or lymphoid) at the initial diagnosis, is converted to the opposite type when it relapses. In recurrent leukemia, lineage switch is rare with an incidence of about 6-9\%. ALL-to-AML is common and most occur in children [1,2]. Common chromosomal abnormalities in lineage switch include translocation of 11q23, chromosome 7 and trisomy 8 [3]. The conversion from AML to ALL is clinically rare [4, 5]. This case report is right about a child converted from AML-M5 to B-ALL.

The pathogenesis of lineage transformation of acute leukemia is not clear. At present, there are three hypotheses: (1) It is mixed lineage leukemia at the first diagnosis, and chemotherapy suppresses the dominant leukemia cloning, resulting in a large number of clones of another phenotype, thus manifested as another type of leukemia [6-8]. (2) The leukemia stem cells have the characteristics of both myeloid and lymphoid progenitor cells at the first diagnosis and have the potential to differentiate into both lineages. This is similar to the blastic phase of chronic myeloid leukemia, which can rapidly turn into acute myeloid leukemia or acute lymphoblastic leukemia [7,9]. (3) Similar to normal hematopoietic stem cells, leukemic cells also have plasticity and reversibility. Under the regulation of many transcription factors or cytokines in the hematopoietic microenvironment, the fate of stem cells may change [10].

After lineage switch, the treatment related to post-conversion phenotype should be selected, but the thera- 
peutic effect of most patients is poor, indicating that the malignancy degree of leukemia with lineage switch is high [8]. At the first diagnosis, the child was diagnosed as AML-M5, relapse as B-ALL and was relieved after treatment with CAR-T therapy. As special cell immunotherapy method, CAR-T therapy has achieved a prospective effect in the treatment of recurrent/ refractory $(\mathrm{R} / \mathrm{R})$ hematological malignancies. Recent studies have found that humanized CD19-CAR-T cells infusion resulted in 2 newly diagnosed B-ALL patients reaching $\mathrm{CR}$, breaking the limitation of CAR- $T$ therapy for $\mathrm{R} / \mathrm{R}$ hematological malignancy [11]. However, this child relapsed again shortly after the remission of CAR-T therapy, underwent chemotherapy and secondary infusion of CAR-T cells, but died from ineffective treatment. Elad et al found that under the persistent immune effect of CAR-T cells targeting antigen CD19, the loss of transcription factors Pax5 and Ebf1 led to the conversion of lineage, which led to the occurrence of resistance to CAR-T immunotherapy [12]. It is further proved that leukemic stem cells are plastic and may undergo lineage transitions due to the influence of the hematopoietic microenvironment. Studies have shown that lineage switch may be more common in immunotherapy such as monoclonal antibodies or CAR-T cells [12-20]. Recent studies have found that nuclear gene rearrangement or epigenetic changes may be related to lineage switch after immunotherapy targeting CD19 $[15,17]$. It has been reported that the B-ALL patient was converted to AML after the treatment with blinatumomab and then converted to B-ALL again after interrupting blinatumomab. This finding suggests that cytokine release is associated with phenotypic changes in leukemia immunotherapy [17]. It is well known that topirazumab (IL-6R signal pathway blocker) can effectively treat lethal cytokine release syndrome (CRS) caused by blinatumomab or CAR-T cells. An early in vitro experiment confirmed that IL-6 can promote the transformation of human leukemia cells to the myeloid lineage. Gardner et al concluded that inflammatory cytokines (mainly IL-6) may cause lymphoid clones to differentiate into myeloid cells. This may partly explain why lineage switch occurs after immunotherapy. Also, Balducci E et al reported a patient who changed from B-ALL to AML after CD19 immunotherapy [14]. In this case, after the child's recurrence, myelocytology still showed a significant increase in primary lymphocytes. Due to the absence of the leukemia immunophenotyping, it is not known whether there is a deletion of targeted molecules such as CD19 or the expression of myeloid antigens. Amandeep et al reported a patient who was diagnosed as T-ALL at the first diagnosis, then relapsed into AML and relapsed again into T-ALL after remission with treatment, which also confirmed the plasticity of leukemic stem cells [13].

This case suggests that the prognosis of acute leukemia with lineage switch after relapse is poor [8, 21]. Treatment needs to be adjusted according to the phenotype after recurrence. If AML is converted to B-ALL and expressing CD19, CAR-T therapy can make it easy to get CR, but prone to secondary recurrence [22]. Clinically, for recurrent leukemia, it is necessary to improve leukemia immunophenotyping, genetic mutation detection, cancer chromosome, and other cytotogenetic and molecular biology examination, so as better to guide the treatment and evaluate the prognosis.

Contribution: Ya-nan Zhang, Feng-an Liu and Xue Wang contributed equally to this work. Xue Wang, Ya-nan Zhang and Feng-an Liu analyzed the data, and wrote and edited the manuscript; Kun-ming Qi and Wei Chen performed some of the analyses. All authors approved the final manuscript.

Conflict-of-interest disclosure: The authors declare no competing financial interests.

Acknowledgments: This work was supported by the National Natural Science Foundation of China (82070127).

\section{CONSENT:}

Written informed consent was obtained from the patient's parents.

\section{References}

1. Paganin, M., et al., A Case of T-cell Acute Lymphoblastic Leukemia Relapsed As Myeloid Acute Leukemia. 2016. 63 (9): p. 1660-3.

2. Della Starza, I., et al., A case of lineage switch from B-cell acute lymphoblastic leukaemia to acute myeloid leukaemia. Role of subclonal/clonal gene mutations. 2016. 174 (4): p. 648-51. 
3. Cuneo, A., et al., Lineage switch and multilineage involvement in two cases of $p H$ chromosome-positive acute leukemia: evidence for a stem cell disease. 1994. 79 (1): p. 76-82.

4. Nomani, L., J. Cook, and H.J.I.j.o.l.h. Rogers, Very rare lineage switch from acute myeloid leukemia to mixed phenotype acute leukemia, B/Myeloid, during chemotherapy with no clonal evolution.2019. 41 (4): p. e86-e88.

5. Hanley, B., et al., Lineage switch from acute myeloid leukemia to T cell/myeloid mixed phenotype acute leukemia: First report of an adult case. 2018. 93 (12): p. E395-E397.

6. Wu, B., et al., Lineage Switch Between B-Lymphoblastic Leukemia and Acute Myeloid Leukemia Intermediated by "Occult" Myelodysplastic Neoplasm: Two Cases of Adult Patients With Evidence of Genomic Instability and Clonal Selection by Chemotherapy. 2017.148 (2): p. 136-147.

7. Gupta, S., et al., Secondary pure erythroid leukaemia in relapsed acute lymphoblastic leukaemia: lineage switch or chemotherapy effect? 2014. 2014.

8. Rossi, J., et al., Lineage switch in childhood acute leukemia: an unusual event with poor outcome. 2012. 87 (9): p. 890-7.

9. Stass, S. and J.J.C.i.h. Mirro, Lineage heterogeneity in acute leukaemia: acute mixed-lineage leukaemia and lineage switch. 1986.15 (3): p. 811-27.

10. Hu, T., R. Murdaugh, and D.J.F.i.o. Nakada, Transcriptional and Microenvironmental Regulation of Lineage Ambiguity in Leukemia.2017. 7 : p. 268.

11. Cao, J., et al., Humanized CD19-specific chimeric antigen-receptor T-cells in 2 adults with newly diagnosed B-cell acute lymphoblastic leukemia. 2019. 33 (11): p. 2751-2753.

12. Jacoby, E., et al., CD19 CAR immune pressure induces B-precursor acute lymphoblastic leukaemia lineage switch exposing inherent leukaemic plasticity. 2016. 7 : p. 12320.

13. Aujla, A., et al., Lineage switch from T-cell lymphoblastic leukemia/lymphoma to acute myeloid leukemia and back to T-cell lymphoblastic leukemia/lymphoma in a patient diagnosed during pregnancy. 2019. 6 : p. 12.

14. Balducci, E., et al., Lineage switch from B acute lymphoblastic leukemia to acute monocytic leukemia with persistent $t(4 ; 11)(q 21 ; q 23)$ and cytogenetic evolution under CD19-targeted therapy.2017. 96 (9): p. 1579-1581.

15. Haddox, C., et al., Blinatumomab-induced lineage switch of B-ALL with t(4:11)(q21;q23) KMT2A/AFF1 into an aggressive AML: pre- and post-switch phenotypic, cytogenetic and molecular analysis. 2017.7 (9): p. e607.

16. Aldoss, I. and J.J.B. Song, KMT2AExtramedullary relapse of ()-rearranged acute lymphoblastic leukemia with lineage switch following blinatumomab. 2018. 131 (22): p. 2507.

17. Wölfl, M., et al., MLLSpontaneous reversion of a lineage switch following an initial blinatumomab-induced ALL-to-AML switch in -rearranged infant ALL. 2018. 2 (12): p. 1382-1385.

18. Oberley, M., et al., Myeloid lineage switch following chimeric antigen receptor T-cell therapy in a patient with TCF3-ZNF384 fusion-positive B-lymphoblastic leukemia. 2018. 65 (9): p. e27265.

19. Rayes, A., et al., Lineage Switch in MLL-Rearranged Infant Leukemia Following CD19-Directed Therapy. 2016. 63 (6): p. 1113-5.

20. Zoghbi, A., et al., Lineage switch under blinatumomab treatment of relapsed common acute lymphoblastic leukemia without MLL rearrangement. 2017. 64 (11). 
21. Fallah Azad, V., et al., $C D 7$ aberrant expression led to a lineage switch at relapsed childhood acute pre- $B$ lymphoblastic leukemia. 2016. 49 (1): p. 53-6.

22. Higuchi, Y., et al., Lineage switch with $t(6 ; 11)(q 27 ; q 23)$ from T-cell lymphoblastic lymphoma to acute monoblastic leukemia at relapse.2016. 209 (6): p. 267-71. 